\title{
Loss Analysis of a 100kW PV Inverter
}

\section{Ruiming YUAN ${ }^{1, a}$, Hengchun DING ${ }^{2, b}$, Jihong QIAN ${ }^{3, c}$, Ying $\mathrm{CHEN}^{4, d}$, Xiaoyu $\mathrm{XI}^{5, \mathrm{e}}$ and Xiaobo $\mathrm{YANG}^{6, \mathrm{f}}$}

${ }^{1}$ Electric Power Research Institute, State Grid JIBEI Electric Power Co., Ltd, Beijing, 100053, China

${ }^{2}$ State Grid JIBEI Electric Power Co., Ltd, Beijing, 100053, China

${ }^{3}$ Wasion Group, Changsha, 410205, China

${ }^{4}$ School of Electrical Engineering, Xi'an Jiaotong University, Xi'an, 710049,China

${ }^{5}$ Electric Power Research Institute, State Grid JIBEI Electric Power Co., Ltd, Beijing, 100053, China

${ }^{6}$ Electric Power Research Institute, State Grid JIBEI Electric Power Co., Ltd, Beijing, 100053, China

ayuan.ruiming@

nc.sgcc.com.cn, bding.hengchun@nc.sgcc.com.cn, c18605677@qq.com, dying.zc.g@gmail.com,

exi.xiaoyu@nc.sgcc.com.cn, ${ }^{\mathrm{f}} 13811909550 @ 163 . c o m$

Keywords: Photovoltaic (PV) Generation System; PV inverter; Power Loss

Abstract. This paper deals with the power loss of a photovoltaic inverter system. The research aims at revealing the loss mechanism of different parts and developing corresponding mathematical calculation methods of the loss. In the first part the structure of a commonly used three-phase grid-connected PV inverter is introduced. The possible losses in this system come from the IGBTs, diodes, capacitor on the DC side, LCL filter, control circuit, cooling system, etc. In the following parts, the losses of each part are analyzed and calculated. Finally, the validity of the proposed calculation methodology is assessed by comparing the theoretical and experimental results.

\section{Introduction}

In recent years, solar energy and other renewable energy sources have attracted much attention and have been developing fast all over the world. In 2010, the total global installations of photovoltaic power plant reached 18.2 GW [1]. And China sees a rapid increase of market share of global solar cell production from 10\% in 2010 to over 59\% worldwide in 2014 [2]. The percentage of electricity produced by PV generation systems is growing and the power loss inside the systems is worth consideration. The loss in a grid-connected PV system mainly consists of photovoltaic array loss, maximum power point tracking (MPPT) loss, DC cable loss, inverter system loss, AC cable loss, etc. In large-scale PV systems these losses can be critical. This paper deals with the inverter system loss.

Inverter system loss mainly consists of loss in the IGBTs and diodes, loss in the DC-side capacitor, loss in LCL filter and other losses. Currently for the first 3 kinds of losses there are various ways to calculate them precisely, but with rather complicated formula and parameters difficult to get. Consequently these calculation methods are not easily implemented in practice.

In this paper, the losses of PV inverter system are first analyzed. A compromise had been made between calculation precision and complexity and, thus, practical methods are selected to calculate the losses while preserving high accuracy. The validity and utility of the proposed loss calculation method are assessed by comparing the theoretical and experimental results for a 100kW PV inverter.

\section{Structure of a Three-phase PV Inverter}

In PV generation systems, the role of PV inverters is to convert the DC power generated by PV cells into AC power. Currently, the inverter system of large-scale PV generation stations mostly take the topology as shown in Fig. 1. 
As can be seen from this topology, the possible losses caused by imperfection of the power devices may come from the IGBTs, diodes, DC-side capacitor, LCL filter, wire, circuit breaker, contactor, etc. The loss of each component will be analyzed and calculated in the following parts.

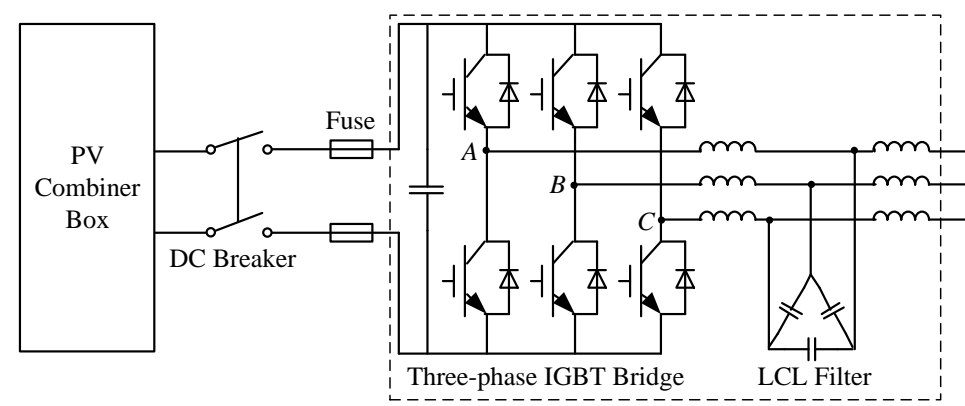

Fig. 1 Diagram of a three-phase PV inverter with LCL filter

\section{Loss Analysis of a Three-phase PV Inverter}

Losses of grid-connected PV inverters mainly come from the semiconductor devices (IGBTs and diodes), DC-side capacitor, LCL filter (inductors and capacitors), and other parts (cooling system, control system, etc.). The model of losses in each part is built and analyzed as follows.

Power electronic device losses. Due to the imperfection of the IGBTs and the diodes, losses exist in real applications of these devices. The losses in these two devices can be divided into 2 categories: switching loss and conduction loss.

1) Switching loss

For IGBTs, switching loss includes the turn-on and turn-off loss. For diodes, it includes the turn-on loss and the reverse-recovery loss. The turn-on loss of the diode, which is much smaller than the reverse-recovery loss, is often neglected in practice.

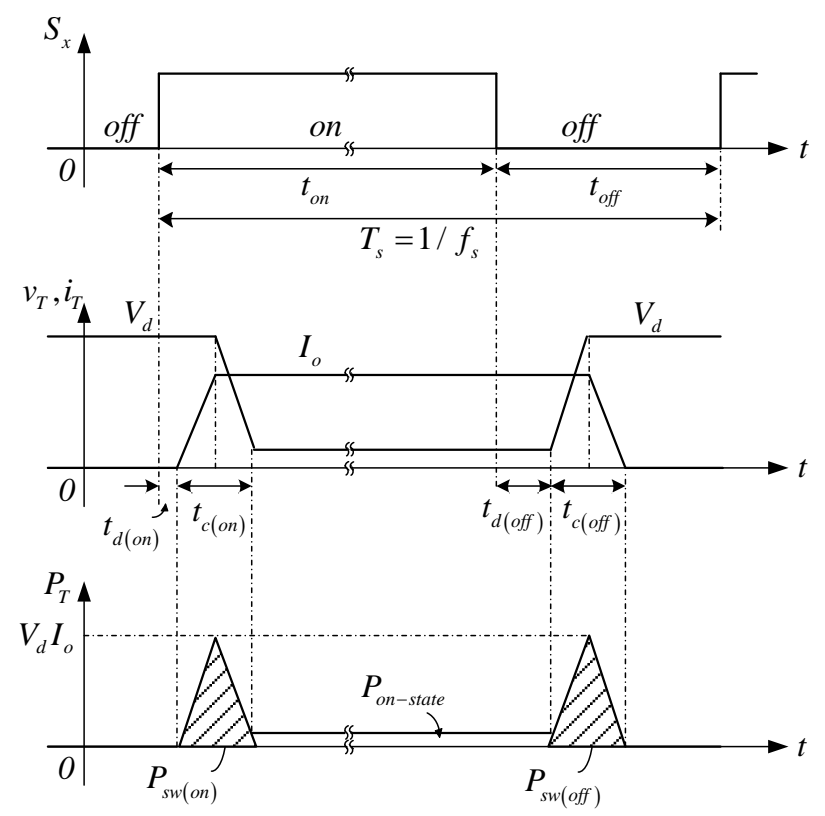

Fig. 2 Losses of an IGBT

Losses of a single IGBT is shown in Fig. 2. When the state of the switch changes, there will be a time delay after the command signal until the completion of the change. Switching losses are generated during these delay periods. For a three-phase PWM inverter with switching frequency $f_{s}$, the switching loss of an IGBT can be calculated by [3]

$$
P_{s w, I G B T}=f_{s} \frac{1+\cos \theta}{2 \pi}\left(E_{\text {on }}+E_{\text {off }}\right) \frac{V_{d}}{V_{C E N}} \frac{I_{0}}{I_{C N}}
$$


where, $V_{C E N}$ is the nominal collector-emitter voltage; $I_{C N}$ is the nominal collector current; $E_{\text {on }}$ and $E_{\text {off }}$ are the turn-on and turn-off energy of a single IGBT with rated voltage and current, respectively; $\theta$ is the phase difference between output voltage and current.

For the diode, only the reverse-recovery loss is considered, consequently the switching loss for a single diode can be calculated by [3]

$$
P_{\text {sw,Diode }}=f_{s} \frac{1+\cos \theta}{2 \pi} E_{\text {off }} \frac{V_{d}}{V_{N}} \frac{I_{0}}{I_{N}}
$$

where, $V_{N}$ and $I_{N}$ are the nominal voltage and current, respectively; $E_{\text {off }}$ is the turn-off energy.

2) Conduction loss

For a three-phase PWM inverter, the conduction loss of a single IGBT, caused by non-zero voltage drop during conduction state, is calculated by [4]

$$
P_{i}=\left(\frac{1}{8}+\frac{M}{3 \pi} \cos \theta\right) r_{T} I_{C M}^{2}+\left(\frac{1}{2 \pi}+\frac{M}{8} \cos \theta\right) V_{F 0} I_{C M}
$$

where, $V_{F O}$ and $r_{T}$ are the forward threshold voltage and resistance of the IGBT, respectively; $I_{C M}$ is the peak current of the inverter output; $M$ is the PWM modulation ratio.

Similarly, the conduction loss for a single diode is

$$
P_{d}=\left(\frac{1}{8}-\frac{M}{3 \pi} \cos \theta\right) r_{D} I_{C M}^{2}+\left(\frac{1}{2 \pi}-\frac{M}{8} \cos \theta\right) V_{D 0} I_{C M}
$$

where $V_{D 0}$ and $r_{D}$ are the forward threshold voltage and resistance of the diode, respectively;

DC-side capacitor loss. By taking into account the loss behavior, the real capacitor can be modeled as an ideal capacitor in series with an equivalent resister (see Fig. 3) [5]. In Fig. 3, Z refers to the total impedance, $C$ is the equivalent series capacitor, $R_{S}$ refers to the equivalent series resistor (ESR), $\delta$ is the dielectric loss angle, $\theta$ is the phase angle between $R_{S}$ and $Z$ (supplementary angle of $\delta$ ), $D$ is the dissipation factor ( $\tan \delta$ ) of capacitor.
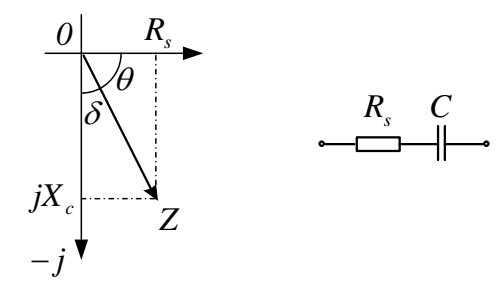

Fig. 3 Equivalent circuit model of a real capacitor

The relationship between these coefficients is

$$
\begin{aligned}
& Z=R_{S}-j X_{C} ; X_{C}=\frac{1}{\omega C}=\frac{T}{2 \pi C} ; \\
& D=\tan \delta=\frac{R_{S}}{X_{C}}=R_{S} \cdot \omega C ; R_{S}=D \cdot X_{C}=\frac{D}{\omega C}=\frac{T \cdot \tan \delta}{2 \pi C}
\end{aligned}
$$

The loss of the capacitor is calculated by

$$
\Delta E_{0}=\int_{0}^{T / 2} R_{S 0} \square(t)^{2} \square d t .
$$

For the capacitor on the DC side, the loss is mainly caused by the current ripple. This current ripple, using SVPWM modulation, is calculated by [6]

$$
\Delta I=I_{m} \sqrt{2 m\left[\frac{\sqrt{3}}{4 \pi}+\cos ^{2} \varphi\left(\frac{\sqrt{3}}{\pi}-\frac{9}{16} m\right)\right]}
$$

where $I_{m}$ is the peak current on the AC side of the inverter, $m$ refers to the modulation ratio of SVPWM, $\varphi$ is the phase difference of the output voltage and current. 
Consequently, by using the equivalent resistance and current ripple, the loss of the capacitor on the DC side can be calculated.

LCL filter loss. For high-power inverters, the often used filter is the $3^{\text {rd }}$ order LCL filter. According to the topology, losses come from the inductors and the capacitors. Generally the losses caused by the inductors are relatively larger than that caused by the capacitors.

1) Capacitor loss

According to the capacitor model shown in Fig. 3 and loss calculation method (Eq. 6), the loss of capacitors on AC-side can be deduced. In this case, the AC current as well as the harmonics should be taken into consideration instead of the current ripple compared to the DC-side capacitor. The loss can be expressed as [5]

$$
P_{C_{-} \text {total }}=C^{2} \sum_{h=1}^{h=h_{\max }} R_{s h} \omega_{h}^{2} U_{h}^{2}
$$

where $h$ refers to the order of the harmonic, $R_{s h}=h R_{s 1}, \omega_{h}=2 \pi f_{h}$, and $U_{h}$ is the rms value of the $h$-th harmonic voltage.

Defining the loss factor $\tan \delta$ as

$\tan \delta=R_{S} \omega C$,

Eq. 8 can be written as

$$
P_{C_{-} \text {total }}=C \sum_{h=1}^{h=h_{\max }}\left(\tan \delta_{h}\right) \omega_{h} U_{h}^{2}
$$

2) Inductor loss

The inductor loss can be divided into two parts: iron loss (core loss) and copper loss (winding loss).

The copper loss is generated by the resistance of the winding and is calculated as [7]

$$
P_{\text {cu }}=R_{\mathrm{ac}} I_{\text {rms }}^{2}
$$

where $R_{a c}$ is the ac resistance, $I_{r m s}$ is the rms value of the current, and practically $R_{a c}$ can be deduced by

$$
R_{\mathrm{ac}}=R_{\mathrm{dc}}\left[1+\frac{\left(r_{o} / \delta\right)^{4}}{48+0.8\left(r_{o} / \delta\right)^{4}}\right]
$$

where $r_{o}$ is the radius of the round conductor and $\delta$ is the skin depth which is calculated by

$$
\delta=\frac{1}{\sqrt{\pi f \mu \sigma}}
$$

with $f, \mu, \sigma$ referring to the wave frequency, permeability of the conductor and conductivity of the conductor, respectively. $R_{d c}$ is the dc resistance and is practically calculated by

$$
R_{\mathrm{dc}}=N(M L T)\left(\rho_{20}\right)\left[1+\alpha_{20}\left(\mathrm{~T}_{\max }-20\right)\right]
$$

where $N$ is the number of winding turns, $M L T$ is the mean length of a turn, $\rho_{20}$ is the dc resistance per centimeter of the material, $T_{\max }$ is the maximum temperature of the device (temperature rise $\Delta T$ plus the ambient temperature $T_{a}$ ). The values of all the parameters can be found in the datasheet of the inductor.

The iron loss, caused by the change of magnetic field in the core, is divided into hysteresis loss, eddy-current loss and anomalous loss. In this case the first loss is much larger than the other two losses, so only the hysteresis loss is considered. It is often calculated by the Steinmetz equation as

$$
P_{\mathrm{fe}}=K_{c} f^{\alpha} B_{\max }{ }^{\beta}
$$

where $f$ is the frequency, $B_{\max }$ is the maximum magnetic intensity; $K_{c}, \alpha, \beta$ are Steinmetz parameters.

However, in power electronic applications, flux waveforms are not sinusoidal due to the switching converters. In this case the Steinmetz equation is no longer precise especially when the switching frequency is higher than $10 \mathrm{kHz}$. The improved generalized Steinmetz equation is thus proposed [7,8] 


$$
\begin{aligned}
P_{v} & =\frac{1}{T} \int_{0}^{T} k_{i}\left|\frac{d B(t)}{d t}\right|^{\alpha}|\Delta B|^{\beta-\alpha} d t=k_{i}|\Delta B|^{\beta-\alpha} \frac{1}{T} \int_{0}^{T}\left|\frac{d B(t)}{d t}\right|^{\alpha} d t \\
& =k_{i}|\Delta B|^{\beta-\alpha}\left|\frac{d B(t)}{d t}\right|^{\alpha}
\end{aligned}
$$

where $\alpha, \beta, k_{i}$ are Steinmetz parameters, and

$$
k_{i}=\frac{K_{c}}{2^{\beta-1} \pi^{\alpha-1} \int_{0}^{2 \pi}|\cos \theta|^{\alpha} d \theta} .
$$

Since the formula is quite complicated, in the considered frequency range still the Steinmetz equation is applied with the precision requirement satisfied, and

$$
B_{\max }=\frac{\Delta B}{2}=\frac{\left(V_{i}-V_{o}\right) D T}{2 N A_{c}}
$$

where $V_{i}$ and $V_{o}$ are the input and output voltage; $D T$ is the turn-on time of the switch; $N$ is the number of turns and $A_{c}$ is the cross-section area of the core.

Other losses. Other parts of the losses include the losses of the control and cooling system, and heat generated in devices such as DC breaker, contactor, and surge arrester when current flows through because of their inner resistance. The first two can be evaluated from datasheets or through experiments, but the other losses are difficult to calculate since the inner parasitic resistance parameters are difficult to evaluate. In practice, these losses are considered constant according to engineering experiences.

\section{Simulation and Experimental Verification}

The losses in the PV inverter system are analyzed in detail in the above parts. In order to verify the proposed loss calculation methodology, measurement results are compared with the calculated ones. Since the loss of each part is difficult to be separated from the experiment results, simulation methods are utilized. In this article the loss of semiconductor devices is evaluated by simulation. Therefore, the verification procedure includes the following two steps:

1) A well-elaborated simulation based on MATLAB/Simulink is performed in order to verify the correctness of the loss calculation method of power electronic devices (IGBTs and diodes). The simulation model includes the real-time measurement of switching and conduction losses of devices and is illustrated in detail in the following parts.

2) The verification of losses in other parts (inductors, capacitors and other parts) is realized by experiment. This is done by comparing the overall calculated loss with the measurement results at different percentages of output power.

The power diagram of the system and the organization of the verification is shown in Fig. 4. A $100 \mathrm{~kW}$ three-phase PV inverter with topology in Fig. 1 is utilized to validate the calculation scheme, and the system parameters are given in Table 1.

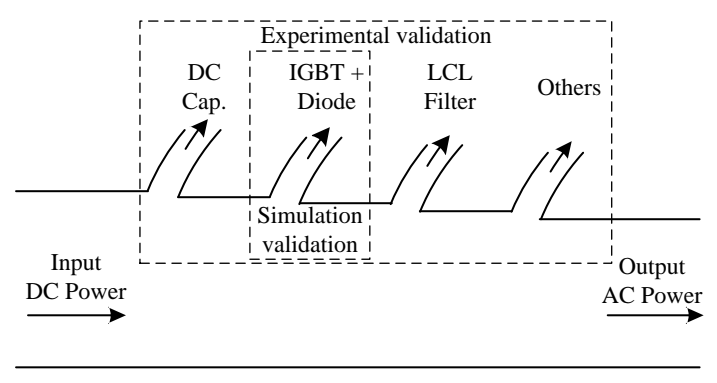

Fig. 4 Power diagram and loss validation

Simulation verification for power electronic devices. Losses of IGBTs and diodes and their calculation methods are considered in this part. The chosen IGBT module is FS300R12KE3 and the input DC voltage is $460 \mathrm{~V}$. 
Table 1 Main parameters

\begin{tabular}{cccc}
\hline Parameter & Value & Parameter & Value \\
\hline$P_{a c, \text { nom }}$ & $100[\mathrm{~kW}]$ & $L_{\text {in }}$ & $0.15[\mathrm{mH}]$ \\
$V_{a c, \text { nom }}$ & $220[\mathrm{~V}]$ & $L_{\text {out }}$ & $0.035[\mathrm{mH}]$ \\
$f_{a c}$ & $50 \pm 0.5[\mathrm{~Hz}]$ & $C_{L C L}$ & $400[\mathrm{uF}]$ \\
$C_{d c}$ & $1.72[\mathrm{mF}]$ & $U_{d c}$ & $330-600[\mathrm{~V}]$ \\
\hline
\end{tabular}

The simulation model is shown in Fig. 1. In the calculation block of the simulation model (see Fig. 5), the switch loss for a single IGBT module is calculated by evaluating the real-time switching and conduction loss and summing these two losses. The look-up tables store the data of the devices from the datasheet, including the $V_{C E}-I_{C}$ curve, the $V_{F}-I_{F}$ curve, $E\left(E_{o n}, E_{\text {off }}, E_{\text {rec }}\right)-I_{C}$ curve, etc.

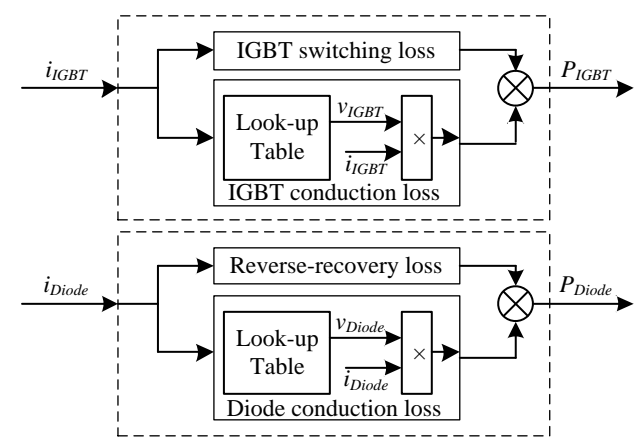

Fig.5 Semiconductor switch loss calculation block of the simulation (for one IGBT module)

The simulations of the IGBT switching loss and the diode reverse-recovery loss are shown in Fig. 6. The logic part discerns the turn-on and turn-on actions of the devices. When a turn-on or turn-off action is recognized, the system checks the look-up table for a correct $\Delta E$ to add to the total loss.

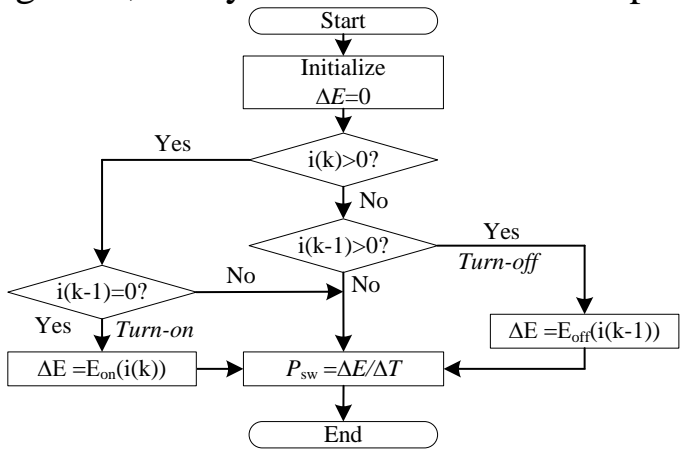

(a) IGBT switching loss

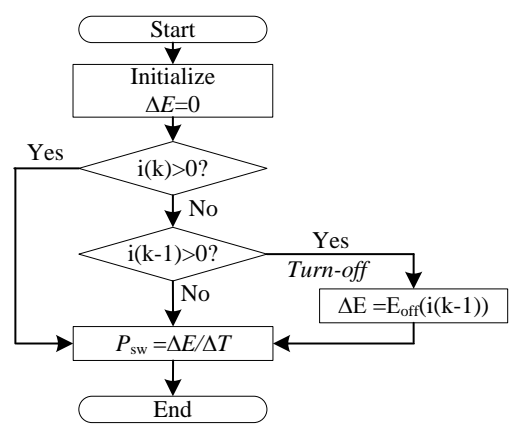

(b) Diode switching loss

Fig. 6 Calculation of switching loss in simulation

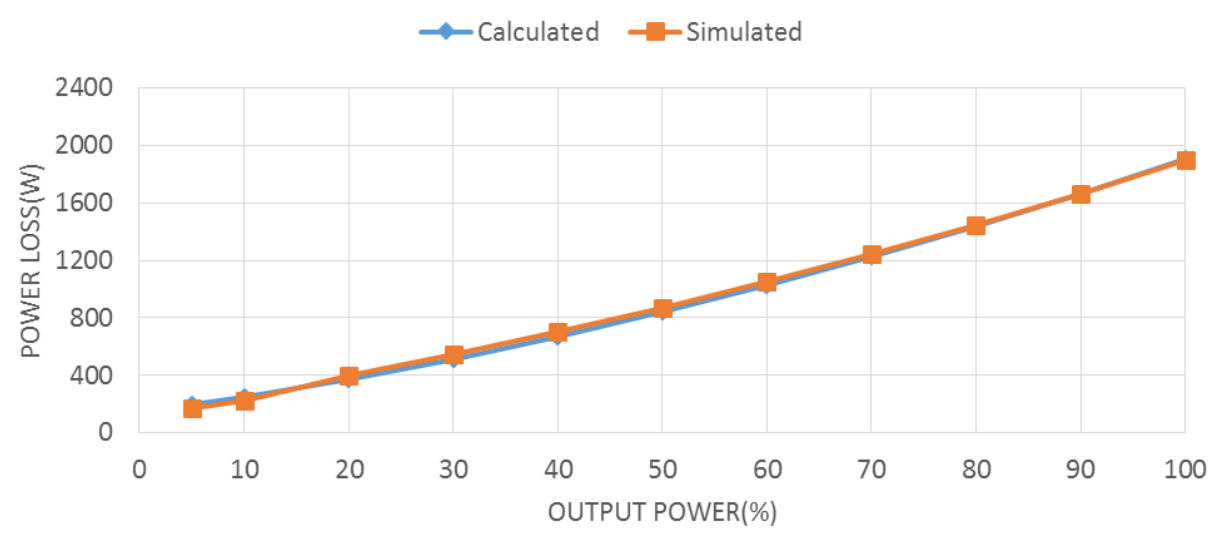

Fig. 7 Power loss of the IGBTs and diodes 
Calculation of the losses of power electronic devices and the real-time simulation of these losses are applied at the same time for comparison. The calculated and simulated power loss of the IGBTs and diodes with different output power are illustrated in Fig. 7.

From Fig. 7, it is apparent that the calculated results have a good matching with the simulated results, at different output power. In this sense, the proposed model of IGBT and diode loss calculation, with its simplicity in the form, can be utilized to calculate the switch loss precisely.

Experiment verification. The topology diagram of inverter system is shown in Fig. 1 and the parameters are given in Table 1. The input and output power of the system at different percentages of rated output power are measured at $40^{\circ} \mathrm{C}$, and the comparison results are shown in Fig. 8 and Fig. 9.

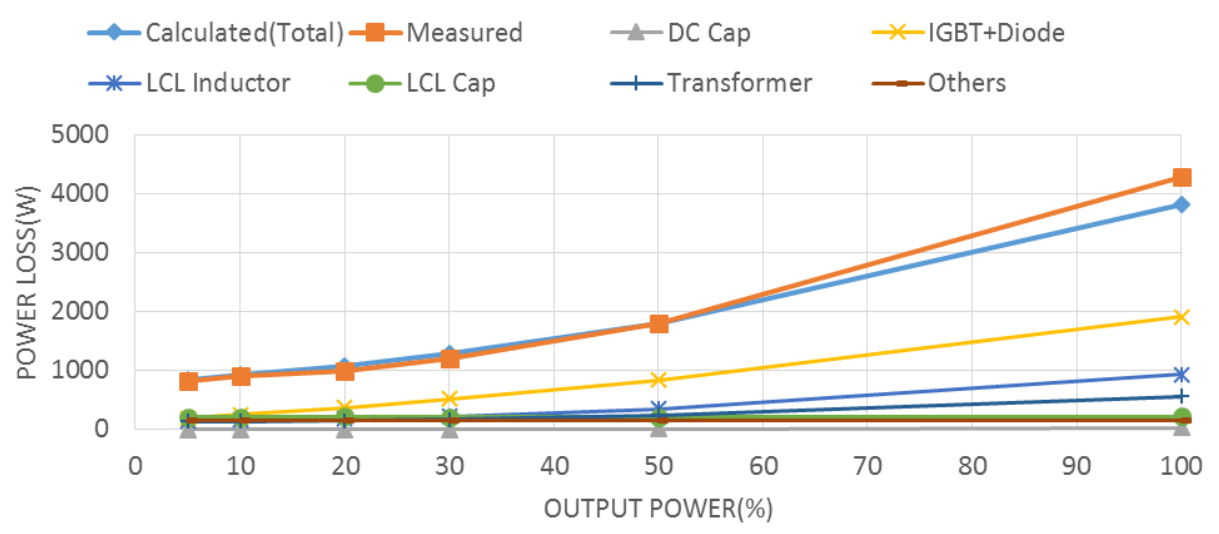

Fig. 8 Overall power loss analysis

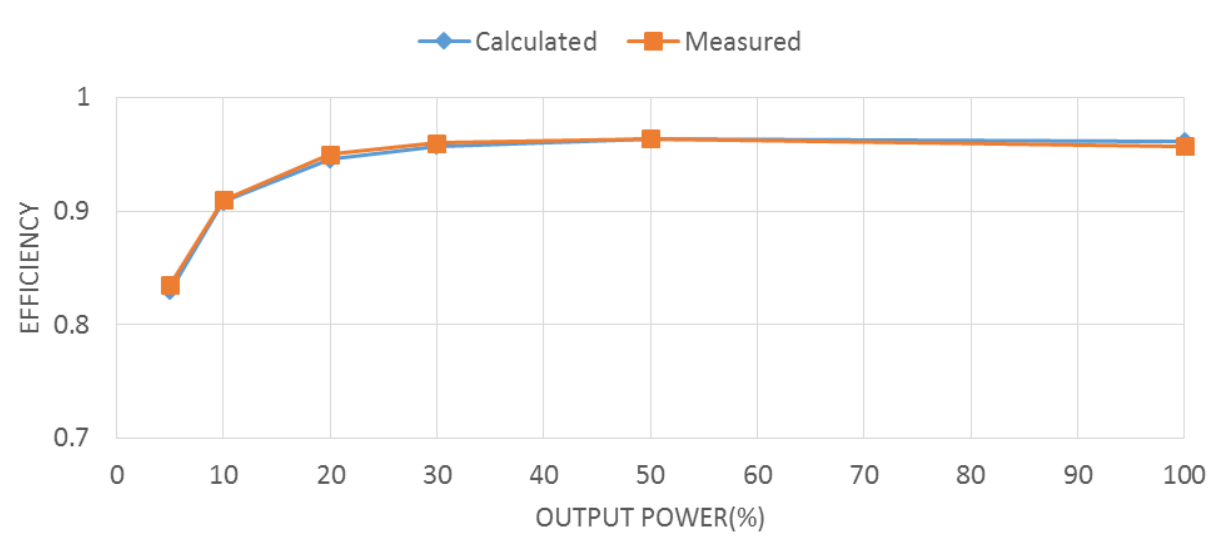

Fig. 9 Efficiency at different output power percentage

From Figs. 8 and 9, it is obvious that the calculated results are precise especially with low output power. When the output power increases to $100 \%$, the error is the largest $(10.7 \%)$. In all the other cases, the error is smaller than $9 \%$. In this sense, the proposed calculation model can be used to calculate and analyze the system loss in a relatively precise way.

Using the calculation results in Fig. 8, the loss of each part can be evaluated and, hence, specific improvements can be harnessed in order to decrease the major losses. This result helps to better design the inverter system with lower loss.

\section{Conclusion}

In this paper, the losses of PV inverter system are analyzed, and practical methods of loss calculation are proposed to evaluate the loss of each component. Simulation and experimental results for a three-phase $100 \mathrm{~kW}$ PV inverter validated the proposed calculation methodology. The result is beneficial for a better understanding of loss mechanisms and for the design of low-loss inverter systems. 


\section{References}

[1] Information on http:// www.solarbuzz.com

[2] Morgan, R. ; SEMI, San Jose, CA, USA ; Weiss, B. ; Berwind, J. ; Raithel, S. The impact of a rapidly changing global PV market on the PV manufacturing supply chain[C], Photovoltaic Specialists Conference (PVSC), 2012 38th IEEE.

[3] LIU Yao. Loss Analysis and Optimal Design of PV Grid-connected Converter [D]. Beijing Jiaotong University, 2011.

[4] Kolar, J.W.; Zach, F.C. Losses in PWM inverters using IGBTs [J], Electric Power Applications, IEE Proceedings -, Jul 1995, 285-288.

[5] Huang, Chaofeng ; Cymer, Inc., San Diego, CA ; Melcher, P. ; Ferguson, G. ; Ness, R. "Loss Estimation of Capacitor in High Rep-Rate Pulsed Power System”. [C] Pulsed Power Conference, 2005 IEEE.

[6] Kolar J W, Wolbank T M, Schrodl M. Analytical calculation of the RMS current stress on the DC link capacitor of voltage DC link PWM converter systems [J]. 1999.

[7] W.G. Hurley, W.H. Wölfle. Transformers and Inductors for Power Electronics: Theory, Design and Applications. ISBN: 978-1-119-95057-8, 370 pages, April 2013.

[8] Shimizu T, Kakazu K, Matsumori H, et al. Iron loss eveluation of filter inductor used in PWM inverters[C]. Energy Conversion Congress and Exposition (ECCE), 2011 IEEE. 606-613. 